WALKer, N. \& Wiltshire, G. H. (1955). J. gen. Microbiol. 12, 478-483

\title{
The Decomposition of 1-Chloro- and 1-Bromonaphthalene by Soil Bacteria
}

\author{
By N. WALKER AND G. H. WILTSHIRE \\ Rothamsted Experimental Station, Harpenden, Hertfordshire
}

SUMMARY: Two species of naphthalene-utilizing bacteria from soil were able to metabolize 1-chloro- and 1-bromonaphthalene. From cultures in which 1-chloronaphthalene was the sole source of organic carbon, D-8-chloro-1:2-dihydro-1:2-dihydroxynaphthalene and 3-chlorosalicylic acid were isolated. 3-Bromosalicylic acid was isolated from cultures with 1-bromonaphthalene and evidence of the formation of a 'diol' compound obtained. The course of the metabolic pathways is discussed.

In previous work, Treccani, Walker \& Wiltshire (1954) studied 5 strains of bacteria, obtained from soil, which could grow with naphthalene as their only supply of organic carbon. Evidence was presented that D-trans-1:2-dihydro1:2-dihydroxynaphthalene, salicylic acid and catechol were successive metabolic intermediates. These organisms also grow in a mineral salts medium with 1-chloronaphthalene as the carbon source; the study of the metabolism of this compound and of 1-bromonaphthalene forms the subject of the present paper. The two organisms which we used in cultures for the isolation of metabolic intermediates are referred to as organism I and organism II. Some of these results have already been reported briefly (Walker \& Wiltshire, $1953 b)$.

\section{METHODS}

Bacteria. Organism I was described by Walker \& Wiltshire (1953a). Organism II was considered by Treccani $e t$ al. (1954) to be a strain of Pseudomonas desmolyticum, Gray \& Thornton (1928).

Media. The mineral salts medium used by Tausson (1927), but adjusted to $\mathrm{pH} \mathrm{6.9,} \mathrm{was} \mathrm{used,} \mathrm{with} \mathrm{addition} \mathrm{of} \mathrm{1-chloronaphthalene} \mathrm{or} \mathrm{1-bromo-}$ naphthalene (both British Drug Houses Ltd. laboratory grade reagents).

Culture conditions. Stock cultures were maintained on plates of Tausson's medium solidified with agar $(2 \%, \mathrm{w} / \mathrm{v})$ and inverted over naphthalene crystals. Cultures in liquid media were grown in $1 \mathrm{l}$. Roux bottles containing $100 \mathrm{ml}$. salts solution and one or two drops of 1-chloro- or 1-bromo-naphthalene. Incubation was usually at $25^{\circ}$. Some cultures were grown at room temperature in $6 \mathrm{l}$. round-bottomed flasks containing $4 \mathrm{l}$. medium stirred mechanically.

Detection of intermediates. The reactions for the detection of chloro- and bromo-naphthalene diols and of chloro- or bromo-salicylic acids were those used by Walker \& Wiltshire (1953 $a$ ) for the corresponding unsubstituted compounds.

Absorption spectra. The absorption spectra of several chloro-naphthols were measured in the range $240-360 \mathrm{~m} \mu$., using a Unicam S.P. 500 spectrophotometer. 


\section{Reference compounds}

4-Chloro-1-naphthol was prepared by the method of Kast (1911).

5-Chloro-1-naphthol was synthesized by the method of Erdmann \& Kirchhoff (1888). It was also prepared from 5-nitro-1-chloronaphthalene (Hodgson \& Walker, 1933) by reduction to 5-chloro-1-naphthylamine followed by diazotization and hydrolysis of the diazonium group.

8-Chloro-1-naphthol was synthesized from 8-nitro-1-naphthylamine (prepared by the method of Hodgson \& Davey, 1939), by replacement of the amino group by chlorine using the procedure of Hodgson \& Walker (1933), followed by reduction to 8-chloro-1-naphthylamine, diazotization and hydrolysis of the diazonium group. (8-Chloro-1-naphthol could not be obtained by the hydrolysis of 8-nitro-1-chloronaphthalene with boiling water as reported by Woroshtzow \& Koslow (1936) nor when water was replaced by dilute aqueous sulphuric acid containing a little urea or by dilute aqueous sodium carbonate.)

8-Chloro-2-naphthol was obtained by replacing the amino group of 7-methoxy1-naphthylamine by chlorine in the usual Sandmeyer procedure and demethylation of the resulting 7-methoxy-1-chloronaphthalene using $48 \%$ aqueous hydrobromic acid (Pope \& Woodcock, unpublished; cf. James \& Woodcock, 1951).

3-Chlorosalicylic acid was synthesized in very small yield by a Kolbe reaction on $o$-chlorophenol.

3-Chloro-2-acetoxybenzoic acid. 3-Chlorosalicylic acid (70 mg.) was heated for $5 \mathrm{~min}$. with a few drops of acetic anhydride and, after cooling, water was added, the mixture heated until clear and then filtered. On standing, the filtrate deposited glistening colourless plates, m.p. 122-123 $^{\circ}$ (18 mg.). (Found: $\mathrm{C}, 50 \cdot 22 ; \mathrm{H}, 3.08 \% ; \mathrm{C}_{9} \mathrm{H}_{7} \mathrm{O}_{4} \mathrm{Cl}$ requires: $\mathrm{C}, 50 \cdot 35 ; \mathrm{H}, 3.26 \%$.)

\section{RESULTS}

\section{Isolation and constitution of the chloronaphthalene diol}

When the diol concentration in Roux bottle cultures of organism I growing on chloronaphthalene was at a maximum (usually after 4-6 days of incubation) the culture fluid was collected, neutralized with aqueous sodium hydrogen carbonate, heated to about $60^{\circ}$ to flocculate the inorganic phosphate precipitate and cells, and filtered; residual chloronaphthalene remained on the filter. To each litre of clear yellow filtrate, $1 \mathrm{~g}$. purified animal charcoal (British Drug Houses Ltd.) was added, and after thorough shaking, the charcoal was filtered off and dried at room temperature; the filtrate was retained for isolation of chlorosalicylic acid (see below). The dried charcoal was extracted for $8 \mathrm{hr}$. in a Soxhlet apparatus with a mixture $(1: 1)$ of ethanol and benzene, and the extract evaporated to dryness under reduced pressure. The residue was dissolved in water, filtered and the solution extracted repeatedly with ether. The combined ethereal extracts were dried $\left(\mathrm{Na}_{2} \mathrm{SO}_{4}\right)$, filtered and concentrated. From the concentrated solution, chloronaphthalene 
diol crystallized out and was recrystallized from benzene in colourless needles, m.p. $108^{\circ},(\alpha)_{\mathrm{D}}^{20^{\circ}}+77^{\circ}, c=0.9$ in EtOH. (Found: C, 60.54; H, 4.6; Cl, 18.17\%; $\mathrm{C}_{10} \mathrm{H}_{9} \mathrm{O}_{2} \mathrm{Cl}$ requires : $\mathrm{C}, 61 \cdot 06 ; \mathrm{H}, 4.6 ; \mathrm{Cl}, 18.07 \%$. $E_{\max .8} .9 \times 10^{-3}$ at $263 \mathrm{~m} \mu$. in $95 \%$ ethanol, $2.0 \times 10^{-3}$ at $293 \mathrm{~m} \mu$. and $1.3 \times 10^{-3}$ at $305 \mathrm{~m} \mu$.)

Constitution of the chloronaphthalene diol. The chloronaphthalene diol behaved like naphthalene diol in being dehydrated to a phenol by boiling for a few minutes with $\mathrm{N}-\mathrm{HCl}$. When the resulting solution was adjusted to pH 10. and treated with $n$-butanol and 2:6-dichloroquinonechloroimide (phenol reagent) a blue colour becoming greener on standing was produced in the butanol layer, but no colour was given without previous acid treatment. The rate of colour development was slightly slower than with 1-naphthol which gives a blue colour. 8-Chloro-1-naphthol behaved like 1-naphthol, but 8-chloro-2-naphthol gave a slowly developing green colour.

The presence of two phenolic compounds after acid treatment of the chloronaphthalene diol was demonstrated by running paper chromatograms, using 0.1N aqueous ammonia as the developing solvent (Boyland \& Sims, 1953). The spots were detected by spraying the paper after drying with a solution of diazotized $p$-nitroaniline followed when dry with aqueous sodium carbonate. As controls, drops of $2 \%$ ethanolic solutions of 8-chloro-1-naphthol, 8-chloro2-naphthol and an equal mixture of the two were used. The $\boldsymbol{R}_{p}$ value of 8-chloro-1-naphthol was slightly greater than that of 8-chloro-2-naphthol. These observations suggested that acid treatment of the chloronaphthalene diol gave a mixture of mainly 8-chloro-2-naphthol with some 8-chloro-1-naphthol.

A similar experiment with $d$-trans-1:2-dihydro-1:2-dihydroxynaphthalene from a naphthalene culture, and from the urine of a rabbit dosed with naphthalene and with solutions of 1- and 2-naphthols as controls, showed that the naphthalene diol gave a mixture of 1-naphthol with a very small proportion of 2-naphthol on acid treatment.

Dehydration of chloronaphthalene diol. The diol (21 mg.) was treated with $4 \mathrm{~N}-\mathrm{HCl}$ on a boiling water bath for $5 \mathrm{~min}$. and the solution concentrated to dryness. The residue $(19.6 \mathrm{mg}$.) was taken up in dilute aqueous sodium hydroxide, the solution filtered and acidified with $\mathrm{HCl}$. The white precipitate that formed was collected, dried and sublimed under reduced pressure at $100^{\circ}$, affording colourless needles, m.p. $97-98^{\circ}$. On admixture with authentic 8-chloro-2-naphthol (m.p. $100^{\circ}$ ) the m.p. was $98-99^{\circ}$. The m.p. was depressed on admixture with 8-chloro-1-naphthol. In another experiment, a yield of $3 \mathrm{mg}$. 8-chloro-2-naphthol (37\% theory) was obtained from $9 \mathrm{mg}$. chloronaphthalene diol by the above procedure.

Supporting evidence of the identity of the chloronaphthol isolated above was obtained by determining its absorption spectrum in the ultraviolet region (240-360 $\mathrm{m} \mu$.). Three distinct zones of absorption, which is a characteristic of 2-naphthols (cf. Daglish, 1950) were distinguished. Comparison with the spectrum of authentic 8-chloro-2-naphthol showed that the spectra were similar, although this evidence is not decisive since 1-chloro-2-naphthol, for example, also has a similar spectrum. The latter compound has a different m.p., $70^{\circ}$ (Cleve, 1888). No other monochloro-2-naphthols were available. 
The spectrum of the reaction mixture from acid-treated chloronaphthalene diol was compared with that of mixtures of 8-chloro-1- and 8-chloro-2-naphthols; it appeared that the reaction mixture contained about $10 \%$ of 8 -chloro1-naphthol. Since the chloronaphthalene diol on treatment with dilute acid yielded 8-chloro-2-naphthol and very probably 8-chloro-1-naphthol, it is concluded that the diol is a dextro form of 8-chloro-1:2-dihydro-1:2-dihydroxynaphthalene.

\section{Isolation of 3-Chlorosalicylic acid}

The filtrate from the charcoal used for adsorbing the chloronaphthalene diol was acidified with dilute $\mathrm{HCl}$ and treated with $1 \mathrm{~g}$. animal charcoal/l. After filtering off, the charcoal was dried and eluted with boiling ethanol or dilute aqueous sodium hydrogen carbonate. In the former case, the ethanol was evaporated and the residue dissolved in aqueous sodium hydrogen carbonate. This solution was then extracted with ether to remove non-acidic material, and the aqueous layer separated, acidified with $\mathrm{HCl}$ and again extracted with ether. This ethereal extract was dried $\left(\mathrm{Na}_{2} \mathrm{SO}_{4}\right)$, filtered and the solvent removed giving crystalline 3-chlorosalicylic acid. This was purified by vacuum sublimation at $120^{\circ}$ and recrystallization from water, affording colourless needles, m.p. $178^{\circ}$, not depressed on admixture with an authentic specimen. (Found: $\mathrm{Cl}, 20.3 \%$; calc. for $\mathrm{C}_{7} \mathrm{H}_{5} \mathrm{O}_{3} \mathrm{Cl}, \mathrm{Cl}, 20.58 \%$.) Addition of aqueous $\mathrm{FeCl}_{3}$ to a dilute solution of the acid gave an intense violet colour. On treatment of the acid with hot acetic anhydride, followed by crystallization from water, a colourless crystalline acetyl compound, m.p. 122-123 ${ }^{\circ}$, not depressed on admixture with authentic 3-chloro-2-acetoxybenzoic acid, was obtained.

\section{Bromonaphthalene diol}

After about 2 or 3 days incubation at $25^{\circ}$ cultures of organism I or II growing on 1-bromonaphthalene gave a weak blue phenol reaction after boiling for $2 \mathrm{~min}$. with $\mathrm{N}-\mathrm{HCl}$. The compound responsible for this test was extracted from neutral aqueous solution by ether, and although it has not been isolated or examined further, it is probably a bromonaphthalene diol.

\section{Isolation of 3-bromosalicylic acid}

Eight Roux bottles containing $125 \mathrm{ml}$. Tausson's medium and 2 drops of 1-bromonaphthalene were inoculated with organism $I$ and incubated at $25^{\circ}$. After 5 days all the cultures were yellow and gave a weak violet ferric reaction. The cultures were collected, concentrated under reduced pressure to about a tenth of their volume, and after acidifying with dilute $\mathrm{HCl}$, were extracted 3 times with ether. The combined ethereal extracts were washed with a little water, then extracted with dilute aqueous sodium hydrogen carbonate. The aqueous layer was separated, acidified with dilute $\mathrm{HCl}$ and extracted with ether. The ether layer was separated, dried $\left(\mathrm{Na}_{2} \mathrm{SO}_{4}\right)$ and the ether removed leaving a brownish solid ( $7 \mathrm{mg}$.) which was crystallized from water (charcoal) giving nearly colourless crystals (4 mg.), m.p. 130-150 ${ }^{\circ}$. The material obtained 
from three such batches of cultures was combined, sublimed in vacuo at $140^{\circ}$, and the sublimate was crystallized from water in colourless needles, m.p. $181^{\circ}$. (Found: $\mathrm{C}, 39 \cdot 41 ; \mathrm{H}, 2 \cdot 375 ; \mathrm{Br}, 36 \cdot 8 \%$ calc. for $\mathrm{C}_{7} \mathrm{H}_{5} \mathrm{O}_{3} \mathrm{Br}: \mathrm{C}, 38 \cdot 71 ; \mathrm{H}, 2 \cdot 305$; $\mathrm{Br}, 36.87 \%$. Müller (1909), found m.p. $184^{\circ}$ for 3-bromosalicylic acid.) Addition of $\mathrm{FeCl}_{3}$ to a dilute solution of the acid produced a deep violet colour.

\section{DISCUSSION}

Of the three possible 1-chloronaphthalene diols, the product isolated from chloronaphthalene cultures was 8-chloro-1:2-dihydro-1:2-dihydroxynaphthalene, the orientation of which was established by dehydration in dilute acid solution to chloronaphthol. In contrast to the formation of 1-naphthol from unsubstituted 1:2-dihydro-1:2-dihydroxynaphthalene (Young, 1947), the chlorinated diol yielded mainly a 2-naphthol. The formation of a small amount of 8-chloro-1-naphthol was shown by paper chromatography and explains the initial appearance of a blue colour, later becoming greenish blue, in the phenol reaction with acid-treated chloronaphthalene diol.

Treccani et al. (1954) found that the metabolism of naphthalene by five different strains belonging to at least three species of soil bacteria followed a similar course. All the strains can also grow on 1-chloronaphthalene, producing a diol and a chlorosalicylic acid, but no chloronaphthol. Diol formation appears to be a general reaction in the oxidation of polycyclic aromatic hydrocarbons, but little is known about the subsequent metabolism of such diols. Their ready dehydration to phenols, and the isolation of phenol conjugates from the urine of mammals dosed with hydrocarbons has led to the belief that both phenols and diols are metabolites, e.g.

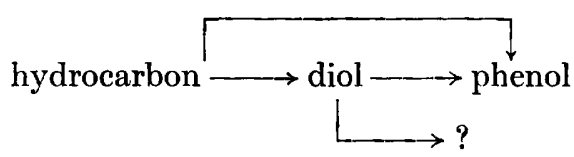

With bacteria there is no evidence that naphthols are formed from naphthalene, and the recent isolation of naphthalene diol glucuronide (Corner, Billett \& Young, 1954) which accounted for nearly all the 'extra glucuronide' in naphthalene-dosed rabbit urine suggests that what had previously been estimated as 1-naphthol glucuronide from animals may well have been diol glucuronide. The evidence from both bacteria and animals favours the conclusion that the diols are true metabolic intermediates, whereas naphthols may be artefacts or at best arise from side-reactions. The intermediate compounds between naphthalene diol and salicylic acid remain to be identified. On this point, the study of the metabolism of 1-chloronaphthalene indicates that: (1) oxidation occurs in the ring not substituted with chlorine, since 8-chloro1:2-dihydro-1:2-dihydroxynaphthalene is the diol formed; (2) the carbocyclic ring so de-aromatized is broken first, giving rise to a chlorosalicylic acid; (3) carbon atom 4 in 8-chloro-1:2-dihydro-1:2-dihydroxynaphthalene becomes the carboxyl carbon atom of 3-chlorosalicylic acid. It is still not known at which" bond opening of the ring occurs. 
Although growth of organism I or II in 1-bromonaphthalene media is slower than with 1-chloronaphthalene, the metabolic pathway is similar, since a diol compound can be detected and 3-bromosalicylic acid accumulates in the cultures. The bromonaphthalene diol has not been isolated, but it seems reasonable to expect this to be 8-bromo-1:2-dihydro-1:2-dihydroxynaphthalene.

We are grateful to Dr D. Woodcock for a gift of 8-chloro-2-naphthol, and to Dr H. G. Thornton, F.R.S., for his interest and encouragement.

\section{REFERENCES}

Boyland, E. \& Sims, P. (1953). The action of some free radicals on naphthalene. J. chem. Soc. p. 2966.

Cleve, P. T. (1888). Über die Einwirkung von Chlor auf $\alpha$ - und $\beta$-Naphtol. Ber. dtsch. chem. Ges. 21, 891.

Corner, E. D. S., Billett, F. S. \& Young, L. (1954). Biochemical studies of toxic agents 6. The conversion of naphthalene into 1:2-dihydro-2-hydroxy-1-naphthylglucosiduronic acid in the rabbit. Biochem. J. 56, 270.

DAGLISH, C. (1950). The ultraviolet absorption spectra of some hydroxynaphthalenes. J. Amer. chem. Soc. 72, 4859.

ErdmanN, H. \& Kirchioff, R. (1888). Die Constitution der isomeren Naphtalinderivate. II. Disubstituirte Naphtaline aus den isomeren Chlorphenylparaconsäuren. Annalen, 247, 366.

Gray, P. H. H. \& Thornton, H. G. (1928). Soil bacteria that decompose certain aromatic compounds. Zbl. Bakt. (2. Abt.), 73, 74.

Hodgson, H. H. \& Davey, W. (1939). Mononitration of $\alpha$ - and $\beta$-naphthylamines in the presence of urea. $J$. chem. Soc. p. 348.

Hodgson, H. H. \& WALKER, J. (1933). The diazotisation of aromatic nitroamines and the prevention of diaryl formation in the Sandmeyer reaction. J. chem. Soc. p. 1620.

JAMES, P. M. \& Woodcock, D. (1951). Synthesis of plant growth regulators. Part 1. Substituted $\beta$-naphthyloxy-acetic acids. J. chem. Soc. p. 3418.

KAST, H. (1911). Chlorierung von $\alpha$-Naphtol. Ber. dtsch. chem. Ges. 44, 1337.

MüLler, J. (1909). Bromoderivate des o-Amido- und o-Oxybenzaldehyds. Ber. dtsch. chem. Ges. 42, 3695.

TAusson, W. O. (1927). Naphtalin als Kohlenstoffquelle für Bakterien. Planta, 4, 214.

Treccani, V., Walker, N. \& Wiltshire, G. H. (1954). The metabolism of naphthalene by soil bacteria. J. gen. Microbiol. 11, 341.

WALKer, N. \& Wiltshire, G. H. $(\mathbf{1 9 5 3} a)$. The breakdown of naphthalene by a soil bacterium. J. gen. Microbiol. 8, 273.

WALKer, N. \& WiLtShire, G. H. (1953b). The decomposition of naphthalene and $\alpha$-chloro- and $\alpha$-bromo-naphthalenes by soil bacteria. Riass. comun. 6th Congr. int. Microbiol. $1,175$.

Woroshtzow, N. N. \& Koslow, W. W. (1936). Studien in der Naphtalin-Reihe V. 1-Nitro-8-chloronaphtalin und 8-Chloronaphtol-(1). Ber. dtsch. chem. Ges. 69, 312.

Young, L. (1947). The metabolic conversion of naphthalene to 1:2-dihydroxynaphthalene-1:2-diol. Biochem. J. 41, 417. 